\title{
BIOGEOGRAFIA: REFLEXÕES SOBRE TEMAS E CONCEITOS
}

\author{
Biogeography: reflections on issues and concepts
}

Biogeografia: reflexiones sobre los temas e conceptos

\section{Sueli Angelo Furlan}

Doutora em Ciências - Programa de Pós-Graduação em Geografia Física - FFLCH-USP. ocente e pesquisadora do Departamento de Geografia -USP. Pesquisadora do Programa de Pós-Graduação em Ciências Ambientais da USP - PROCAM. Coordenadora do NUPAUB - USP (Núcleo de Estudos de Populações Humanase Áreas Úmidas (www.usp.br/nupaub). Pesquisadora da United Nations Research Institute for Social Development- UNSRID (Suiça) (www.unrisd.org). Especialista em Planejamento Ambiental. Av. Prof. Lineu Prestes, 338. CEP. 05508-000 - São Paulo - SP-Brasil.

Email:sucaangf@usp.br /suelifurlan@uol.com.br

\section{Rosemeri Melo e Souza}

Doutora em Desenvolvimento Sustentável/Gestão Ambiental (UnB) com estágio doutoral Grupo DILIF da Universidade de Lisboa, Portugal. Pós-Doutora em Geografia Física (Biogeografia) pela GPEM/The University of Queensland, Austrália. Pesquisadora do CNPq e Professora Associada do Departamento de Engenharia Ambiental da Universidade Federal de Sergipe (UFS). Docente Pesquisadora dos Programas de Pós-Graduação em Geografia (PPGEO/UFS) e em Desenvolvimento e Meio Ambiente (PRODEMA/ UFS). Lidera o Grupo de Pesquisas GEOPLAN/UFS - Geoecologia e Planejamento Territorial (www. geoplan.net.br). Av. Marechal Rondon S/N, Pólo de Pós-Graduação, sala 01. CEP: 49100-000 - São Cristóvão-SE-Brasil.Email: rome@ufs.br

\section{Eduardo Rodrigues Viana de Lima}

Doutor em Geografia - Programa de Pós-Graduação em Geografia - Universidade Estadual Paulista Júlio de Mesquita Filho (UNESP). Pesquisador do Programa de Pós-Graduação em Geografia e do Programa de Pós-Graduação em Desenvolvimento e Meio Ambiente da Universidade Federal da Paraíba. Pós-doutorado na Universidad de Sevilla (Espanha). Bolsista de Produtividade em Pesquisa do CNPq - Nível 2 - Arquitetura, Demografia, Geografia, Turismo e Planejamento Urbano e Regional. Universidade Federal da Paraíba, Centro de Ciências Exatas e da Natureza - Campus I, Departamento de Geociências. Cidade Universitária- Castelo Branco III - CEP58059-900 - João Pessoa - PB - Brasil. Email:eduvianalima@gmail.com

\section{Bartolomeu Israel de Souza}

Doutor em Geografia pela Universidade Federal do Rio Grande do Sul (UFRGS). Pós-Doutorado em Biogeografia na Universidad de Sevilla (Espanha). Professor dos Programas de Pós-Graduação em Geografia e Desenvolvimento e Meio Ambiente da Universidade Federal da Paraíba. Coordenador do Grupo de Pesquisa em Estudos do Semiárido - GESA (UFPB). Universidade Federal da Paraíba, Centro de Ciências Exatas e da Natureza, Departamento de Geociências - UFPB/CCEN/Departamento de Geociências, Campus I. Cidade Universitária, Castelo Branco III, CEP: 58059-900, João Pessoa/PB, Brasil.Email: bartolomeuisrael@gmail.com 


\section{RESUMO}

Este artigo traz uma breve análise das produções científicas apresentadas no XI Encontro Nacional da Anpege de 2015. Pela primeira vez organizou-se um eixo com o título Biogeografia e Geoecologia da Paisagem, revelando que a pesquisa sobre a dimensão espacial da biodiversidade vem ampliando sua produção e espaços de divulgação acadêmica em eventos da Geografia. Destacam-se também as bases conceituais e metodológicas das pesquisas e a apropriação de tecnologias de geoprocessamento nas análises biogeográficas. A paisagem e os geossistemas aparecem como conceitos estruturadores de variados métodos de estudo. Neste contexto apresenta-se uma caracterização do campo de conhecimento da Biogeografia na Geografia e algumas articulações conceituais com outros campos científicos. Em seguida analisa-se a amostra de trabalhos que demonstram esses recortes temáticos e enfoques que atestam, particularmente, o dinamismo da disciplina na contemporaneidade. Por outro lado, percebe-se uma certa diluição dos conteúdos de estudos biogeográficos em vários temas da Geografia Física. Outro aspecto importante é o papel das abordagens espaciais e cartográficas, assim como aquelas que sugerem um novo foco geográfico da conservação da natureza. As análises confirmam a necessidade de uma sistematização, ainda que preservando a riqueza e diversidade de tendências, pois ao que parece tem-se neste momento tangências evidentes em vários campos disciplinares que sempre dialogaram com a biogeografia. Saberes de campos científicos, tais como: Botânica, Zoologia, Ecologia da Paisagem, entre outros, estão presentes nos estudos que objetivam de modo geral compreender a espacialização dos seres vivos. Além destes campos, que podem ser considerados externos ao campo específico da Geografia para interpretação biogeográfica, conclui-se que é imprescindível entrelaçar e explorar o grande potencial da Geografia como um dos suportes fundamentais para o estudo de questões ambientais, associadas principalmente com a Geografia da Conservação.

Palavras-chave: biogeografia, geografia da conservação, paisagem.

\section{ABSTRACT}

This article provides a brief analysis of the scientific papers presented at the XI Anpege National Meeting of 2015. For the first time organized a Biogeography session and this shows that research on the spatial dimension of biodiversity has increased its production and spaces academic disclosure in Geography events. Also noteworthy is the conceptual and methodological basis of research and ownership of GIS technology in the biogeographical analyzes. The landscape and geosystems appears as a key concept of different methods of study. In this context presents a characterization of Biogeography field of knowledge in geography and some conceptual links with other scientific fields. Then analyzes the sample of works that demonstrate these thematic approaches prove particularly the dynamism of discipline nowadays. On the other hand, one sees a certain dilution of the contents of biogeographical studies on various topics of Physical Geography. Another important aspect is the role of spatial and cartographic approaches, as well as those that suggest a new geographic focus of nature conservation. The analysis confirms the need for systematization is to preserve the richness and diversity of trends, because it seems we have this tangency evident when 
several disciplines that always dialogued with biogeography. Knowledge of scientific fields, such as botany, zoology, Landscape Ecology, among others, are present in studies that aim to generally understand the spatial distribution of living beings. In addition to these fields, which can be considered external to the specific field of Geography for biogeographical interpretation, it is concluded that it is essential interlacing and explore the great potential of geography as one of the main supports for the study of environmental issues associated mainly Conservation Geography.

Keywords: biogeography, conservation geography, landscape.

\section{RESUMEN}

Este artículo proporciona un breve análisis de la producción científica presentada en el XI Encuentro Nacional Anpege de 2015. Es el primer equipo quién organizó el eje con el título Biogeografía y esto demuestra la investigación que en la dimensión espacial de la biodiversidad ha aumentado su producción y espacios académicos de divulgación en eventos de Geografía. También es destacable la base conceptual y metodológico de la investigación y la apropiación de la tecnología SIG en los análisis biogeográficos. El paisaje y Geosystems aparece como un concepto de estructuración de los diferentes métodos de estudio. En este contexto se presenta una caracterización del campo de Biogeografía de los conocimientos de geografía y algunos enlaces conceptuales con otros campos científicos. Luego se analiza la muestra de obras, sus recortes temáticas y enfoques que lo que demuestra especialmente el dinamismo de la disciplina en la actualidad. Por otro lado, se ve la dilución de los contenidos de los estudios biogeográficos sobre diversos temas de la geografía física. Otro aspecto importante es el papel de los enfoques espaciales y cartográficos, así como aquellos que sugieren un nuevo enfoque geográfico de la conservación de la naturaleza. El análisis confirma la necesidad de sistematización a preservar la riqueza y la diversidad de tendencias, ya que parece que tenemos esta tangencia evidente con varias disciplinas que siempre dialogaron con la biogeografía. El conocimiento de los campos científicos, como la botánica, la zoología, la ecología del paisaje, entre otros, están presentes en los estudios que intentan comprender en general, la distribución espacial de los seres vivos. Además de estos campos, que pueden considerarse externas al campo específico de la Geografía para la interpretación biogeográfica, se concluyó que es esencial explorar el gran potencial de la geografia, uno de los principales soportes para el estudio de los problemas ambientales asociados principalmente Geografia de la Conservación.

Palabras clave: biogeografía, geografía de la conservación, paisaje. 


\section{INTRODUÇÃO}

A Biogeografia é um campo de conhecimento essencialmente interdisciplinar. Compartilha conceitos e métodos de vários outros campos científicos, mas distingue-se deles por apresentar um conjunto de questões próprias, tais como: $\mathrm{O}$ que condiciona as diferentes espacialidades dos seres vivos nas paisagens? Como explicar a diversidade da vida e sua organização espacial? Como explicar as fragmentações históricas e contemporâneas de habitats? Como tempo e espaço se combinam nos arranjos dos seres vivos? Quais escalas de análise são adequadas para explicar esses arranjos? Como explicar as transformações que ocorreram em muitas épocas da história da vida no planeta Terra? Como compreender as mudanças ambientais que vem ocorrendo e os novos arranjos da biodiversidade na época contemporânea? Como entender que existem diferentes modos de interpretar uma formação vegetal? Como aplicar os conhecimentos sobre a espacialidade da vida na conservação da natureza? O que é biogeografia da conservação? Como a biogeografia se fundamenta em preceitos da Geografia da Conservação? Qual o papel da cartografia e das geotecnologias no campo da Biogeografia?

Muitas outras questões poderiam ser acrescidas a esta modesta lista de perguntas, mas não se pretende respondê-las neste artigo, e sim levantar ideias sobre a abrangência, a diversidade de questões e as especificidades que envolvem os estudos biogeográficos. Observa-se, no entanto, que estes questionamentos sugerem a interação entre conhecimentos detalhados da biota e sua dimensão espacial. Ou seja, este é o grande desafio biogeográfico, lidar com espacialidades e temporalidades múltiplas da vida. Como campo de estudo interdisciplinar, uma grande diversidade de temas relativos a espacialidade dos seres vivos é enfocada, mas os desafios de conservação da natureza vêm sendo priorizados nas temáticas da contemporaneidade, dado o estado de degradação e riscos de extinção. Neste sentido uma definição que se coloca como pano de fundo é a formulada por Brown \& Lomolino (2006):

"A Biogeografia é um campo de conhecimento interdisciplinar da Geografia e das Ciências Biológicas que pesquisa o modo como os seres vivos se distribuem no tempo e no espaço". "Éo estudo da biodiversidade espacializada".

Mas como desenvolver os estudos em direção a esta compreensão? Não é tarefa simples construir respostas para as perguntas biogeográficas, pois para compreender a espacialização da biodiversidade é preciso reunir informações distintas e complexas de múltiplos campos do conhecimento, tais como da Botânica, Zoologia, Ecologia, Antropologia, Biologia da Conservação, entre outros. Além dos conteúdos, que podem ser considerados externos 
ao campo específico da Geografia, é imprescindível relacionar a espacialidade dos seres vivos com os aspectos geoecológicos do ambiente e o modo como as sociedades humanas vêm transformando a os sistemas naturais, interferindo na dinâmica da biodiversidade. Portanto, a Biogeografia não é um campo exclusivo da Geografia, mas acrescenta aos estudos da espacialização da biodiversidade conteúdos próprios de sua preocupação analítica sobre a relação sociedade e natureza, da qual se destacam os estudos sobre espacialização da sociodiversidade, compreendendo-a como dinâmica interagente com a biodiversidade.

Por esse motivo, outra questão fundamental tem sido considerar a Biogeografia um dos suportes fundamentais para o estudo de questões socioambientais, principalmente quando se analisa as complexas interações entre Sociedade e Natureza no mundo urbano ou rural, nas políticas públicas de proteção da paisagem, enfim, nos dilemas de conservação. Portanto, a Biogeografia abarca conhecimentos fundamentais para a Conservação da Natureza.

A conservação da natureza tem levado os estudos biogeográficos a um papel central na fundamentação teórica, conceitual e nas práticas do planejamento da proteção da paisagem. Como planejar a proteção das biotas em sua espacialidade considerando as temporalidades naturais e as territorialidades humanas sobrepostas na paisagem? Como entender e propor desenhos consistentes de conservação para as biotas remanescentes de um processo de intensa fragmentação? Como conservar os fragmentos em ambientes humanizados? Como respeitar a sociodiversidade das comunidades tradicionais que colaboram com a conservação da biodiversidade? Estas perguntas necessitam de informações obtidas por múltiplos métodos e isto não se faz sem o domínio da associação de conceitos a um instrumental teórico e analítico específicos da Biogeografia e suas múltiplas combinações científicas. Afinal, os seres vivos se organizam no espaço diante de suas capacidades e limitações e a partir do tempo profundo (GOULD, 1987). Então, como definir áreas de ocorrência e distribuição dos organismos? Como interpretar o arranjo espacial numa floresta tropical, nas restingas, nas dunas costeiras e manguezais, ou nos altiplanos andinos?

Tomando apenas a componente biodiversidade, percebe-se que as perguntas biogeográficas dependem do que se conhece da biota analisada e, muitas vezes, apesar do objetivo ser revelar as funcionalidades dos habitats, tem-se que recuar e iniciar os primeiros passos dos estudos das temporalidades e espacialidades da biota pelos seus inventários, levantamentos empíricos, formação de coleções, etc. Ou seja, o próprio conteúdo da Biogeografia a coloca diante de métodos muito variados, a depender do estado de conhecimento que se tem de uma biota. Vê-se ainda nas produções biogeográficas brasileiras estudos fundamentais realizados como primeiros passos da conservação, que é o levantamento, inventário e mapeamento de ocorrência das espécies. 
Porém, com a disseminação de tecnologias informacionais, a Biogeografia ganhou novas possibilidades de análise espacial. As modelagens espaciais a partir da produção de dados robustos, tem auxiliado na visão abrangente da espacialidade da biodiversidade (DE SIQUEIRA E DURIGAN, 2007; RODRIGUES, 2012; ARASATO E AMARAL, 2013; TAMBOSI et al, 2013, entre outros).

Este processo de disseminação tecnológica tem propiciado novos olhares e novas abordagens acerca dos arranjos espaciais e componentes da(s) Biogeografia(s) pretérita(s), bem como realizar projeções robustas e confiáveis, a partir de diversos algoritmos computacionais, projetando cenários futuros, tanto para domínios biogeográficos como para espécies.

Tais ferramentas metodológicas voltadas para pesquisas em diversidade biológica têm ganhado cada vez mais espaço no universo da ciência e gerado resultados determinantes para investigações científicas. Ferramentas de modelagem de distribuição de espécies, Species Distributions Modelling (SDM), por exemplo, são utilizadas pelos cientistas com o propósito de prever a distribuição potencial de espécies (FRANKLIN; MILLER, 2009).

De modo geral, como afirmam Phillips e colaboradores (2006), um modelo distributivo descreve a adequação no espaço ecológico, normalmente projetada em um espaço geográfico, produzindo uma área geográfica com provável ocorrência de espécies. Logo, áreas que satisfazem o nicho fundamental representam o potencial de distribuição das espécies e as áreas geográficas realmente habitadas correspondem à distribuição realizada.

Os modelos distributivos de espécies confirmam a sensibilidade dos ambientes e/ou, quando este for o foco, das espécies-alvo da modelagem biológica diante de mudanças ambientais. Ressalte-se que quando as condições ambientais, principalmente as climáticas, alteram-se, a vegetação da área atingida também sofre modificações e isto é claramente apurado e refletido através da seleção das camadas ambientais preconizadas nos modelos (SANTOS; ARAÚJO; SOUZA,2015).

Os estudos da espacialização da biodiversidade têm se tornado fundamentais para compreender o dinamismo de uma paisagem. Por isso, tem sido igualmente importante associar a perspectiva teórica do desenvolvimento de capacidades inerentes ao planejamento ambiental. Nesse sentido, a Biogeografia requer o desenvolvimento de habilidades em cartografia, em estatística espacial, em informática, em modelagem espacial, em técnicas de campo voltadas a observar, registrar, comparar, analisar, interpretar e construir as representações e explicações sobre a distribuição dos seres vivos. 


\section{A PAISAGEM, 0 GEOSSISTEMA E A PRÁXIS BIOGEOGRÁFICA}

Estudos da paisagem na práxis biogeográfica têm sido enfatizados pela Ecologia de Paisagens, considerada por alguns autores como seu segundo nascimento, uma vez que o termo teria sido proposto por Troll na Alemanha em1939 (METZGER, 2001). A abordagem recente da Ecologia de Paisagens foi impulsionada a partir da década de 1980, nos Estados Unidos, por biogeógrafos e ecólogos que procuravam relacionar as discussões sobre a relação dinâmica entre área e espécies da Teoria de Biogeografia de Ilhas. Essas ideias, muito discutidas nos últimos 30 anos, têm como um dos seus objetivos, propor desenhos consistentes para o planejamento de áreas protegidas.

A Ecologia de Paisagens trabalha com modelagens espaciais da biodiversidade. As análises espaciais se beneficiaram muito do desenvolvimento das tecnologias digitais, particularmente das imagens de satélite e das análises geoestatísticas, desenvolvendo técnicas avançadas para a delimitação e relação espacial entre os chamados elementos da paisagem (SARTORELLO, 2014; GASPARETTO, 2014).

Em biogeografia este é um dos enfoques que vem se expandindo e ganha força pelo tipo de "análise objetiva que proporciona, atendendo às necessidades de tomadas de decisão para a conservação da diversidade biológica e manejo de recursos naturais, e também pela grande difusão de tecnologias digitais que emanam das empresas norte-americanas. No Brasil, em particular, reflete-se também em políticas públicas como por exemplo em programas como o Biota FAPESP e o Pacto da Mata Atlântica no Estado de São Paulo" (SARTORELLO, op. cit.).

Mas, ao mesmo tempo em que se sofisticam os recursos tecnológicos e métodos geoestatísticos, observa-se uma simplificação da paisagem como totalidade biogeofísica e cultural. Os estudos geográficos da paisagem tentam, por outro lado, abranger um grande número de variáveis biofísicas integradas nos estudos geossistêmicos, mas que encontram grandes barreiras para a aplicação de problemas práticos devido à dificuldade de elaboração da abordagem proposta.

Diante dessas breves considerações, verifica-se que os trabalhos apresentados no XI Encontro da Anpege mostram algumas destas tendências e permanências, sintetizadas em algumas perguntas:

1. Qual a importância da permanência dos estudos baseados em técnicas de inventário (florísticos ou faunísticos)?

A resposta é bastante óbvia. Mesmo com as avançadas técnicas indiretas de análise por meio dos recursos tecnológicos, ainda são fundamentais os levantamentos de dados 
primários sobre a ocorrência e distribuição das espécies. Análises da estrutura da vegetação, o mapeamento de pontos das espécies, os diagramas estruturais, são técnicas muito úteis na biogeografia de campo. Os estudos de campo com métodos e técnicas clássicas são fundamentais e insubstituíveis. Neste sentido, a Biogeografia compartilha procedimentos e técnicas da Botânica e da Zoologia.

2. As relações solos, relevo, vegetação fazem sentido no escopo da Biogeografia contemporânea?

A Biogeografia ecológica estuda as interações entre estes fatores. Os estudos das interações são a chave do entendimento da espacialidade dos habitats, por essa razão as pesquisas integradas em geografia física auxiliam a responder perguntas importantes da Biogeografia aplicada a conservação. A relação área x espécie depende do conhecimento integrado do meio físico. As conexões de habitat dependem de compreensões sobre a funcionalidade de fragmentos das biotas no sentido de ligar as espécies aos demais componentes estruturantes da paisagem. Os estudos de geomorfologia, de solos e de microclima são essenciais para entender conexões funcionais, assim como a abordagem integrada dos geossistemas.

3. A cartografia geossistêmica dialoga com a biogeografia?

A espacialização da biodiversidade é concretizada nos mapas. Isto depende de escalas e integração de fatores. A pesquisa por bio e geoindicadores parece ser outro eixo fundamental para sustentar análises de conservação da natureza. A complexidade do estudo geossistêmico exige do pesquisador uma sólida formação geográfica. O principal desafio tem sido os métodos de integração das variáveis. Venturi (2004) salienta que a dimensão da paisagem implica em se caracterizar uma área de estudo em qualquer escala de trabalho, desde que possa ser aceita na ciência geográfica. Isto implica afirmar que a cartografia aplicada aos estudos da biodiversidade comporta uma dimensão ecológica e, ao mesmo tempo, a possibilidade de articular e registrar múltiplas transformações dos arranjos espaciais no tempo mediante a realização dos estudos dinâmicos e de cenários. Costa (2010) salienta que a cartografia ambiental tem que considerar as relações espaço-temporais, uma vez que terá de defrontar-se com a complexidade da realidade. Este aspecto integrador dos resultados da pesquisa em geo e bioindicadores e suas representações cartográficas, ainda merece ser melhor equacionado no âmbito da Cartografia geossistêmica em seu diálogo com a Biogeografia. 
4. Qual o papel dos estudos experimentais na biogeografia?

Dados primários sobre as interações e processos ecológicos serão sempre fundamentais para compreender a espacialização dos seres vivos. Neste sentido, uma forte aproximação com os estudos bioclimáticos é essencial. Esta é uma vertente que se deve apostar. Aqui poderíamos ampliar para o componente solos que é fator determinante e limitante para as espécies e que pode ser estudado por protocolos experimentais em campo.

5. O que as modelagens espaciais e a biogeografia revelam?

Esta parece ser a chave científica do momento, pois as modelagens da biodiversidade vêm se tornando consistentes e há muito trabalho por fazer. Particularmente as testagens dos desenhos de conservação precisam ganhar densidade em todos os biomas brasileiros. Estudos de processos como a dispersão entre fragmentos no presente e no passado podem ajudar a sugerir melhores políticas de proteção da paisagem que não sejam apenas restritas ao modelo de ilhas das Unidades de Conservação previstas no SNUC (Lei 9985/2000). Pode-se caminhar efetivamente para uma visão de planejamento ecoregional.

Essas questões emergiram da amostra de pesquisas analisadas e revelam que as tendências e permanências nos estudos biogeográficos são aqueles que tentam compreender os diferentes padrões de distribuição dos animais e plantas, seja através de estudos de inventários, protocolos experimentais, modelagem de nichos e da biodiversidade. Reconstruir padrões, unindo a história da Terra em diferentes escalas espaciais e temporais, à história das formas dos seres vivos, é entender como se processaram as modificações dos habitats, quais suas causas e como isso aparece refletido no espaço geográfico. Nesse sentido, todas as opções de pesquisa ocorrem dentro da ótica biogeográfica que requer a observação, coleta de dados, utilização de técnicas que permitam a obtenção de dados primários ou secundários e análises em ambientes informacionais. $\mathrm{O}$ trabalho em Biogeografia se concentra na observação, registro e coleta de informações sobre os componentes da paisagem combinando fatores do meio natural, considerando as escalas de tempo e espaço.

O conteúdo teórico e conceitual enfatiza abordagens múltiplas voltadas a conhecer a biota para desenvolver a Biogeografia aplicada à conservação da natureza e na formulação de políticas públicas, particularmente o planejamento da conservação da natureza. A reflexão que se faz permite propor algo mais abrangente e é importante compartilhar algumas das ideias ainda em gestação. 


\section{BIOGEOGRAFIA E GEOGRAFIA DA CONSERVAÇÃO}

Em que pese a importância de todas as biogeografias que convivem no momento, ainda não se percebem produções voltadas a uma visão integradora do olhar das espécies sobre a paisagem (considerando sua dinâmica, historicidade e fragmentação), e a paisagem das territorialidades da cultura. Não se trata de negar a grande necessidade e repercussão das produções ecológicas, mas ampliar essas produções incorporando métodos integradores de variáveis tanto do meio físico como cultural. Trata-se de ligar a biogeografia à dimensão cultural. Alguns autores já iniciaram esse caminho quando analisaram os traslados de espécies ao longo dos séculos de interação dos homens com os habitats (SIMMONS, 1982), ou das marcas culturais indígenas na distribuição de espécies nativas (POSEY, 1995). Essas bases também aparecem na Biologia da Conservação.

É inegável a importância da conservação da natureza diante da extensiva degradação ambiental, e as ações para que as estratégias sejam efetivas são trabalhosas e se apóiam em diferentes matrizes conceituais e teóricas de distintas áreas disciplinares. Trata-se de um conhecimento complexo. Isto ocorre porque é preciso levar em consideração necessidades complexas da espacialidade da biodiversidade e das populações humanas que co-habitam nos mesmos territórios, explorando os recursos de modo distinto e conflitivo em sua historicidade. A espacialidade dos habitats e a concorrente territorialidade humana é o aspecto central da conservação. Garay \& Becker (2006) apontam que para diferentes espacialidades têm-se diferentes significados que precisam ser pactuados na conservação da natureza. Este vem sendo o enfoque de estudos sobre a conservação ambiental no âmbito da Geografia. Após décadas de produções acadêmicas em diversas áreas do conhecimento objetivando avançar em proposições sobre o tema da Conservação da Natureza, um número crescente de autores têm se dedicado a elucidar a funcionalidade dos habitats naturais através de conceitos e métricas da Ecologia de Paisagens. De outra parte, estudos geográficos demonstram a necessidade de ampliar os estudos de funcionalidade considerando as dimensões físicas totais do meio (SARTORELLO, 2014). Além disso, as espécies percebem os ambientes, assim como segmentos culturalmente diferenciados da sociedade usam e protegem recursos biológicos que exploram em distintos territórios.

Tem-se buscado cientificamente um plano eficiente de conservação biológica, centrado no mosaico das paisagens e na funcionalidade das paisagens para os organismos biológicos (METZGER, 2003). No entanto, percebe-se cada vez mais o aprofundamento de uma visão estritamente biológica, não incorporando adequadamente práticas sociais de comunidades cujas culturas em sua projeção espacial posssuem dimensões igualmente importantes na conservação da natureza, tornando difícil a execução de modelos ou mesmo reduzindo a 
análise espacial. As comunidades que convivem há séculos com as florestas tropicais, por exemplo, desenvolveram uma maneira própria de manejar os recursos, mediante regras socioculturais que colaboraram com a manutenção da biodiversidade e em muitos casos interferiram em sua espacialidade (POSEY, 1995; 1996, BALLÉ, 1994; FURLAN, 2005). Nesse entendimento, a dimensão cultural carece ser articulada aos conceitos funcionais ecológicos. Assim, como existem variáveis funcionais da biota, pode-se trabalhar com a ideia de variáveis da territorialidade da cultura que conectam o modo de ser, viver e trabalhar aos habitats, ambos interagindo na paisagem e jogando com seus atributos de modo articulado.

Em Geografia, o tema da proteção da paisagem não aparece isolado da cultura, particularmente do modo como as sociedades se inscrevem nas paisagens. A leitura da paisagem está, de certo modo, presente em diferentes enfoques das pesquisas sobre as territorialidades. No Brasil há interesse crescente em pesquisas relacionadas a Áreas Protegidas que buscam configurar os melhores desenhos de proteção da paisagem, considerando articuladamente aspectos da Ecologia de Paisagens e da Geografia Cultural. Ambos enfoques são rigorosos quanto ao reconhecimento da importância da manutenção dos habitats, mas valorizam práticas das comunidades indígenas e das populações tradicionais. Neste sentido, buscam uma abordagem colaborativa para construir desenhos que integrem natureza e cultura, entendendo a paisagem como fato cultural, produto inalienável da experiência humana com os habitats. É neste sentido que uma Geografia da Conservação da Natureza poderá se articular com a Ecologia de Paisagens, e os enfoques biogeográficos se ampliam.

De onde emerge essa singularidade na proposição de uma Geografia da Conservação, associado com a biogeografia? Será apenas mais uma expressão? Como se pode desenvolver essa ideia?

A despeito dos enfoques filosóficos diferentes, ao longo da história o pensamento geográfico sempre abordou a relação homem x natureza, ou sociedade x meio ambiente. Esta constante aproximação entre os processos da natureza e a historicidade das sociedades humanas está presente desde a origem da geografiacomo ciência no século XIX. A abordagem ambiental, num primeiro momento dos estudos geográficos, partiu da descrição das paisagens naturais decompondo-as em seus elementos, tais como: clima, relevo, solo, vegetação, hidrografia, flora e fauna. Nesse início, o Homem aparece de forma dissociada desse quadro natural, mas a produção histórica do saber geográfico desenvolveu diversas leituras da paisagem, incluindo a abrangência cultural não alienada dos processos naturais, passando por muitos campos de conhecimento da geografia, buscando integrar os processos do meio geofísico e ecológico às perspectivas históricas da sociedade (MAZZEI, 2007). 
Nas descrições iniciais, cada elemento foi sendo desdobrado e paulatinamente decifrado em seus processos. Pode-se dizer que, parte do arcabouço das ideias geográficas da proteção da paisagem partiu do entendimento destes desdobramentos analisados, a partir das escalas de abordagem, através da visão espacial e das inter-relações decorrentes de interpretações das variáveis naturais e seus comportamentos espaciais. Estas, agrupadas ou integradas conforme conjuntos sistêmicos de suporte, forma e cobertura, foram sendo interpretadas por diferentes percursos de pesquisa, incluindo neste arcabouço os conhecimentos sintéticos da Biogeografia. A impressão humana na paisagem, também tratada como derivações dos sistemas naturais, gerou a necessidade de compreender a espacialidade da biota a partir dos tensores culturais. Evidentemente, não se pretende negar a eficiência dos muitos métodos de análise da paisagem, pois é fundamental compreender como a biota "vê" a paisagem, mas o foco nos organismos a partir da paisagem derivada e valorizada pela sociedade não pode ser redutor e valorizar exclusivamente os atributos funcionais dos organismos.

Defende-se que é preciso avançar e contribuir para somar com a alta eficiência dos modelos fornecidos pela Ecologia de Paisagens e construir desenhos consistentes de conservação para os organismos, incluindo um novo pool de conceitos socioecológicos não rivais da conservação biológica, para buscar complexizar a visão de proteção da paisagem incluindo a dimensão da cultura. Não se trata de pender para uma visão antropocêntrica que nega a importância da funcionalidade dos habitats, pelo contrário, trata-se de trazer à luz matrizes de conceitos que ajudem a entender a dinâmica socioambiental e colaborar para uma visão mais completa da conservação, explicitando conteúdos analíticos da geografia igualmente seguros para a conservação ambiental.

Trata-se de desvendar processos territoriais que influem na efetiva conservação dos sistemas naturais analisados a partir de um pool de conceitos que articulem funcionalidades biológicas e culturais. Sempre que se examinam esses processos focando na dimensão das espécies, não se dissocia das dinâmicas sociais que potencializam, inibem ou excluem a biodiversidade.

\section{GEOGRAFIA DA CONSERVAÇÃO DA NATUREZA: UMA PROPOSTA}

No mundo as paisagens são derivadas, ou seja, são informadas pela cultura. Portanto, considerar a funcionalidade da cultura nesta derivação é uma importante estratégia para melhor gerir o espaço.

É nesta perspectiva que uma Geografia da Conservação pode desenvolver uma abordagem integradora em Biogeografia. Existem caminhos e abordagens teóricas possíveis e serão destacados preliminarmente dois pontos: 
1. Conexões funcionais da biota e conexões culturais impressas na paisagem transformada.

Ampliar o pool conceitual para não dissociar da paisagem sua componente histórica e cultural é necessário. Compreender as relações entre conservação biológica e territorialidades históricas é igualmente desafiador. A título de exemplo destacam-se as territorialidades dos povos tradicionais. Parte-se do pressuposto da importância de abarcar a funcionalidade da cultura e contemplar o COMPLEXO biogeográfico; entrelaçar campos de conhecimento de espacialidades múltiplas das práticas culturais e dos processos ecológicos.

As territorialidades são produzidas historicamente a partir de aprendizagens dos povos com seu meio e, portanto, uma aprendizagem no habitat. Para buscar compreender a territorialidade deve-se investir na reconstrução histórica da formação da paisagem, pesquisando a formação territorial das paisagens na dimensão cultural.

Os territórios atuais que guardam importantes remanescentes da biodiversidade são o resultado de processos históricos. São paisagens informadas pela cultura que contém diferentes camadas do tempo biológico e sociocultural. A conservação destes territórios vividos e ocupados na maioria dos casos por povos tradicionais (indígenas, quilombolas, caiçaras, sertanejos, caboclos e muitos outros) se inscreve em diferentes tempos. Por exemplo, a atual localização de terras indígenas no Brasil é decorrente das pressões promovidas pelas frentes de expansão e não abrangem a territorialidade histórica. Gomide (2010), analisando um corredor de sustentabilidade de matas ciliares em terra Xavante, afirma que...

"Aos povos indígenas restou o refúgio em áreas que não ofereciam os recursos cobiçados pela sociedade abrangente (SEEGER \& CASTRO, 1979: 103-106).Hoje parte da sociedade abrangente quer enquadrar o modo de ser e viver dos povos indígenas em pressupostos da conservação, reconhecendo que suas práticas sociais são mais amigáveis a proteção da biodiversidade quando compradas a outras práticas sociais das sociedades que se sobrepuseram aos seus territórios e exterminam aspectos fundamentais do habitat de muitas espécies."(Gomide, op. cit.).

Mas será suficiente desenvolver a sustentabilidade de um território ao aplicar conceitos da Ecologia da Paisagem a territórios indígenas atuais apenas pelo "olhar" das espécies? O modo como os povos ancestrais exploraram o habitar pode "reforçar" o seu valor como prática conservacionista. É preciso somar as confirmações ecológicas às territorialidades que conectam a natureza aos usos que estas sociedades diferenciadas fizeram e fazem dos recursos naturais. As territorialidades dos povos da tradição se fundamentam numa leitura socioecológica do habitat.

Neste sentido, seriam as métricas da paisagem suficientes para unir as práticas sociais dos povos indígenas às demandas das espécies? O que conserva os territórios do modo 
de ser e viver dos povos indígenas precisa ser confrontado com princípios da ecologia de paisagem para serem validados como prática conservacionista? O ponto de vista é o de que os conceitos stricto sensu não explicam as mesmas coisas, apesar de colaborarem entre si para que se tenha uma melhor conservação e proteção da paisagem em seus aspectos funcionais. A funcionalidade biológica ganharia com o entendimento da funcionalidade cultural e a cultural que poderia também ser iluminada pelo entendimento das conexões imprescindíveis da biota. Em muitos casos os territórios da funcionalidade biológica não são suficientes para abarcar as necessidades territoriais dos povos indígenas e tão pouco das espécies se for desconsiderada a cultura dos povos (CRUZ, 2014; FERREIRA, 2012).

Como então incorporar a territorialidade dos povos tradicionais, cujas práticas sociais são importantes no cenário de fragmentação ambiental imposta pela lógica econômica que capitaliza todos os espaços para finalidades não conservacionistas, e que suprime a funcionalidade de outras culturas mais harmônicas com os ecossistemas?

Incorporar as territorialidades de outros povos, cujas práticas permitem uma paisagem permeável à biodiversidade é vantajoso e pode ser feita se partir de conceitos e métricas adequadas. Quais seriam as trocas importantes mantidas pela prática cultural? Qual seria a extensão destas práticas? Poderia se falar em uma conexão entre formas de usos associadas à dupla espacialidade: da natureza e da cultura? Há espacialidade da vida associada à cultura?

O contraste do uso dos recursos naturais conflita e gera uma situação de risco para as espécies biológicas e também à sobrevivência do modo de vida dos povos indígenas e outros segmentos da sociedade que convivem de modo a articular-se com a dinâmica dos recursos ecossistêmicos nativos, tais como os quilombolas, caiçaras, seringueiros, entre outros. As políticas para a conservação da sociodiversidade, assim como da biodiversidade, deveriam se aproximar e não antagonizar-se.

2. A área de ocorrência e distribuição das espécies e a Biogeografia na Conservação Geográfica.

Um segundo conceito bastante antigo da Biogeografia é a Área de distribuição. Aliás, a ÁREA é um dos mais antigos conceitos da Geografia. Em Biogeografia este conceito é um dos primeiros fundamentos. A Área de ocorrência e distribuição de um organismo é um fundamento essencial do habitat na espacialidade da biodiversidade e, portanto, também fundamental para a conservação. Pois a conservação tem uma localização e a paisagem é a sua extensão. Nos estudos biológicos a área é também o habitat e nicho ecológico ou a extensão da paisagem onde ocorre a interação da espécie com o ambiente. Nos estudos de ecologia de paisagens a área pode ser também fragmento. A área é essencialmente tridimensional e sistêmica, onde vários fatores se ajustam e se modificam mutuamente. 
Na Geografia da Conservação a Área tem uma dimensão sistêmica, evolutiva e cultural. Do ponto de vista das espécies a área é a expressão do sucesso da espécie na exploração dos recursos que compõem o habitat. Do ponto de vista cultural a área é o fragmento utilizável dos recursos, é uma leitura do habitat no campo dos valores. A área, portanto comporta o tempo da natureza e o tempo acumulado historicamente. O tempo da cultura humana e sua capacidade técnica de explorar os tempos da natureza. Analisa-se na atualidade essa sobreposição assimétrica do tempo: o tempo profundo das espécies e o tempo histórico cultural das sociedades humanas. Para pensar um desenho de área de conservação não é possível abstrair a junção de ambos os tempos, pois ele está impresso na paisagem e moldou em camadas a sua forma à sua expressão complexa. Portanto, mesmo se reduzindo a paisagem a sua funcionalidade biológica é preciso incorporar as camadas dos tempos culturais. Para isso o inventário histórico da área, e uma etnografia da área são fundamentais. Uma metodologia retrospectiva do meio físico tem sido proposta em estudos de Geomorfologia antropogênica (RODRIGUES, 2005;2010). É possível buscar uma reconstrução socioecológica da área. Entretanto, em Biogeografia essa retrospectiva ainda é parcial. Algumas análises em Ecologia da Paisagem abstraem o tempo e analisam a paisagem do presente, destituída da história da área.

Em relação à dinâmica territorial da área, tem sido fundamental a modelagem da fragmentação de habitats e conectividade com técnicas de geoprocessamento. A fragmentação no habitat é, sem dúvida, um dos principais impactos da dinâmica das biotas. Seus efeitos refletem principalmente a quebra de continuidade de habitats; ruptura de populações biológicas antes contínuas em populações isoladas ou parcialmente isoladas; processos de extinção de populações locais vêm sendo objeto do grande esforço dos estudos em Ecologia da Paisagem e Biologia da Conservação.

As análises abordam a área e sua configuração territorial a partir de fatores que afetam a diversidade nos fragmentos de habitat em múltiplas variáveis: tamanho dos fragmentos; distância entre os fragmentos, distribuição espacial dos fragmentos, mobilidade dos indivíduos entre os fragmentos, geometria dos fragmentos, efeitos da distância sobre a funcionalidade do habitat, efeito de percolação, permeabilidade, ocupação e presença de espécies em fragmentos a partir de métodos de estudos geoestatísticos. Estes estudos elucidaram uma grande variedade de efeitos biofísicos, tais como mudanças microclimáticas, extensão do efeito de borda, favorecimento das espécies oportunistas e generalistas em detrimento das especialistas, redução da diversidade, mudança na composição da biota, entre muitos outros aspectos. Neste sentido, a Área permanece como conceito-ponte fundamental na análise biogeográfica e que elucida a funcionalidade biológica da conservação. Mas os fragmentos são também produtos de porções do habitat 
valorizados pelos usos da terra que a ocupação humana gerou e sua permanência muitas vezes se dá por esse processo, seja porque é fonte de recursos que não foram suprimidos, proteção humana, legislações, entre outros.

Como afirmou Ab'Saber (1982), existe a necessidade de se apresentar de forma integrada os principais atributos naturais que interagem, acompanhados sempre do maior número de fatores antrópicos que respondam pelo padrão de uso e ocupação dos espaços em estudo, mesmo que estes estudos tratem de um nível de integração preliminar, sujeitos a aperfeiçoamento e detalhamentos. Neste sentido, a matriz de uma paisagem é uma herança de tempos acumulados, como já foi explanado. Para a Geografia da Conservação os modelos de Ecologia de Paisagens poderiam ser a carta base para as análises dos atributos de integração socioecológica, acrescidos da tridimensionalidade da área quanto a seus aspectos geomorfológicos, pedológicos, climatológicos, antropológicos, entre tantos outros da complexidade geográfica.

A despeito dos grandes avanços que a Ecologia da Paisagem vem empreendendo e apontando numa direção consistente para as abordagens biogeográficas, percebe-se que essa abordagem carece de aportes geográficos diversificados e abrangentes, incluindo análises que ampliem as modelagens com parâmetros consistentes do meio físico, ou introduzindo a análise histórica do meio cultural. Neste artigo enfocam-se alguns destes aspectos que se tem trabalhado em Biogeografia, sem adjetivá-la de cultural, pois no âmbito de uma conservação geográfica no sentido de aproximar conceitos e influir na conservação da natureza, propõe uma transversalidade de conceitos geográficos que permitiriam adequar e aproximar a perspectiva da geografia da conservação à perspectiva da ecológica da paisagem e da Biogeografia. Considera-seque as idéias, mesmo que ainda provisórias, devem ser lançadas para que sejam socializadas, debatidas, criticadas e desenvolvidas, assim como ocorreu com a paisagem na dimensão ecológica.

\section{REFERÊNCIAS}

3. AB'SABER, Aziz N. The paleoclimate and paleoecology of Brazilian Amazonia. Biological Diversification in the Tropics. 1982: 41-59.

4. ARASATO, Luciana S. e AMARAL, Silvana. Geoprocessamento e Biodiversidade: contribuições para a modelagem da distribuição de palmeiras Amazônicas. Anais XVI Simpósio Brasileiro de Sensoriamento Remoto - SBSR, Foz do Iguaçu, PR, Brasil, 13 a 18 de abril de 2013, INPE.

5. BALLÉ, Willian. Footprints of the Forest: Kảapor Ethnobotany-the Historical Ecology of Plant Utilization by an Amazonian People. New York: Columbia University Press, 1994 
6. GARAY, Irene e BECKER, Bertha. As Dimensões Humanas da Biodiversidade. O desafio de novas relações sociedade-natureza no século XXI/ Irene Garay e Bertha K. Becker. Petrópolis: Editora Vozes, 2006.

7. BERTRAND, G. Paisagem e geografia física global: esboço metodológico. Caderno de Ciências da Terra, 13: 11-27, 1972.

8. BROWN, James; LOMOLINO, Mark V. Biogeografia. Tradução Julio Feliciano Afonso; revisão técnica Adler Guilherme Viadana. Ribeirão Preto - SP. Funpec Editora, 2006.

9. CRUZ, Takumã M. S. Etnoecologia de paisagens na Terra indígena IramaLaklãnõ, Santa Catarina. Brasil. Dissertação mestrado - Programa de Pós-Graduação em Ecologia da Universidade Federal de Santa Catarina, 2014.

10. DARBY, H.C. The Chaning English Landscape. In: ENGLISH, P. W. \& MAYFIELD, R. C. Man, Space and Environment. New York: Oxford University Press, 1972.

11. DE SIQUEIRA, Marinez F. e DURIGAN, Giselda. Modelagem da distribuição geográfica de espécies lenhosas de cerrado no Estado de São Paulo. Revista Brasil. Bot., V.30, n.2, p.233243, 2007.

12. ENGLISH, P. W. \& MAYFIELD, R. C. Man, Space and Environment. New York: Oxford University Press, 1972.

13. FERREIRA, Marco T. da S. Ecologia histórica aplicada à gestão ambiental comunitária da terra indigna Maxakali, Minas Gerais. Dissertação de mestrado - Programa de Conservação e Manejo da Vida Silvestre - UFMG, 2012, 177p.

14. FORMAN, R.T.T. \& GODRON, M. Landscape Ecology. John Wiley, New York, 1986.

15. FRANKLIN, J. \& MILLER, J.A. 2009. Mapping species distribution: spatial inference and prediction. Cambridge University Press, New York, USA. 2009

16. FURLAN, Sueli A. Florestas culturais: manejo sociocultural, territorialidades e sustentabilidade. Revista AGRÁRIA, São Paulo, No3, pp. 3-15, 2006

17. GASPARETO, Tadeu da Costa. Pressão urbana e conectividade da paisagem no entorno dos parques estaduais de Itapetinga, Itaberaba, Cantareira, Juquery e Jaraguá na região metropolitana de São Paulo. 2014. Dissertação (Mestrado em Geografia Física) - Faculdade de Filosofia, Letras e Ciências Humanas, Universidade de São Paulo, São Paulo, 2014. Disponível em: <http://www.teses.usp.br/teses/disponiveis/8/8135/tde-29042015-143428/>. Acesso em: 2016-04-25.

18. GOMIDE, Maria L. , 2010 GOMIDE, Maria Lucia Cereda. MarãnãBödödi - a territorialidade Xavante nos caminhos do Ró. 2008. Tese (Doutorado em Geografia Física) - Faculdade de Filosofia, Letras e Ciências Humanas, Universidade de São Paulo, São Paulo, 2009. Disponível em: <http://www.teses.usp.br/teses/disponiveis/8/8135/tde-20102009-163712/>. Acesso em: 2016-04-25.

19. GOULD, Stefhen J. Seta do tempo, ciclo do tempo. São Paulo: Cia das Letras, 1987.

20. LANG, Stefan. Análise da Paisagem com SIG. São Paulo, Oficina de Textos, 2009. 
21. LONWENTHAL, David and PRINCE, Hugh C. English Landscape Tastes. .In: ENGLISH, P. W. \& MAYFIELD, R. C. Man, Space and Environment.New York: Oxford University Press, 1972.

22. MAZZEI, Katia, 2007 MAZZEI, Katia. Corredores de fauna na região Cantareira-Mantiqueira: evidências geográficas. 2007. Tese (Doutorado em Geografia Física) - Faculdade de Filosofia, Letras e Ciências Humanas, Universidade de São Paulo, São Paulo, 2007. Disponível em: <http:// www.teses.usp.br/teses/disponiveis/8/8135/tde-11122007-094508/>. Acesso em: 2016-04-25.

23. METZGER, Jean P.O que é ecologia de paisagem? v. 1, n1/2. Biota Neotropica, Campinas, São Paulo, v1, n1/2. 2001.

24. METZGER, Jean P. Effects of slash-and-burn fallow periods on landscape structure. Environmental Conservation, Cambridge, v. 30, n.4, p. 325-333, 2003.

25. MIKESSEL, Marvin W. Landscape. In: ENGLISH, P. W. \& MAYFIELD, R. C. Man, Space and Environment. New York: Oxford University Press, 1972.

26. PHILLIPS Phillips, S.J., R.P. Anderson \& R.E. Schapire. 2006. Maximum entropy modeling of species geographic distributions. Ecological Modelling 190: 231-259

27. POSEY, Darrell A. Indigenous peoples and traditional resource rights: a basis for equitable relationships? Oxford: Green College Centre for Environmental Policy and Understanding.

28. POSEY, Darrell A. Provisions and mechanisms of the Convention on Biological Diversity for Access to Traditional Technologies and Benefit Sharing for Indigenous and Local Communities Embodying Traditional Lifestyles. (OCEES Research Paper, 6). Oxford: Oxford Centre for the Environment, Ethics \& Society. ISBN 1-900316-05-6

29. RODRIGUES, Cleide. Morfologia Original e Morfologia antropogênica na definição de Unidades espaciais de planejamento urbano: exemplo na metrópole paulista. Revista do Departamento de Geografia, 17 (2005) 101-111.

30. RODRIGUES, Cleide. Avaliação do impacto humano da urbanização em sistemas hidrogeomorfológicos. Desenvolvimento e aplicação de metodologia na grande São Paulo. Revista do Departamento de Geografia, 20 (2010) 111-125

31. RODRIGUES, Elisangela S. da C. Teoria da Informação e Adaptatividade na Modelagem de Distribuição das Espécies. Tese de Doutorado em Sistemas Digitais. Escola PolitécnicaUSP, e 2012, 137p.

32. ROUGERIE, G. e BEROUTCHACHVILI, N.L. Géosystèmes et paysages. Bilan et méthodes. Paris: Armand Colin, 1991.

33. SANTOS, Sindiany S. C.; ARAÚJO, Edilson D. de; SOUZA, Rosemeri M. Cenários da distribuição potencial de mangues no litoral norte e nordeste brasileiro a partir da modelagem de distribuição potencial de espécies. Okara: Geografia em debate vol. 9, N. 2 (2015) Edição Especial - I Simpósio Internacional de Biogeografia de Campo 
34. SARTORELLO, Ricardo. Interações em estudos para conservação: conceitos e técnicas para análises geográficas e ecológicas da paisagem. 2014. Tese (Doutorado em Geografia Física) - Faculdade de Filosofia, Letras e Ciências Humanas, Universidade de São Paulo, São Paulo, 2014. Disponível em: <http://www.teses.usp.br/teses/disponiveis/8/8135/tde-24032015122734/>. Acesso em: 2016-04-25.

35. SIMMONS, G. de I. Biogeografia Natural y Cultural. Publisher Omega, 1982

36. SEEGER, Anthony; DA MATTA, Roberto; CASTRO, Eduardo B. V. A construção da pessoa nas sociedades indígenas brasileiras. Boletim do Museu Nacional, Série Antropologia, 1979, n. 32, p. 2-19.

37. TAMBOSI, Leandro R.; MARTENSEN, Alexandre C.; RIBEIRO, Milton C.; METZGER, Jean P. Identificação de áreas para o aumento da conectividade dos remanescentes e unidades de conservação da Mata Atlântica. In Mapeamentos para a conservação e recuperação da biodiversidade na Mata Atlântica: em busca de uma estratégia espacial integradora para orientar ações aplicadas. Brasília: MMA, 2013. 216 p.: il. color.; 29,5 cm. + DVD. (Série Biodiversidade, 49) VENTURI, Luis Antonio Bittar. A dimensão territorial da paisagem geográfica. Anais do VI Congresso Brasileiro de Geógrafos - AGB, Goiânia, 2004. 11 p.

Artigo recebido em 16 de junho de 2016.

Artigo aceito em 16 de julho de 2016. 